\title{
COMMUTING AUTOMORPHISMS OF SOME FINITE GROUPS
}

\author{
S. Fouladi AND R. Orfi
}

Kharazmi University and Arak University, Iran

\begin{abstract}
Let $G$ be a group. An automorphism $\alpha$ of $G$ is called a commuting automorphism if $x x^{\alpha}=x^{\alpha} x$ for all $x \in G$. We denote the set of all commuting automorphisms of $G$ by $\mathcal{A}(G)$. Moreover a group $G$ is called an $A C$-group if the centralizer of every non-central element of $G$ is abelian. In this paper we show that $\mathcal{A}(G)$ is a subgroup of the automorphism group of $G$ for all finite $A C$-groups, $p$-groups of maximal class, and metacyclic p-groups.
\end{abstract}

\section{INTRODUCTION}

Let $G$ be a group and $\operatorname{Aut}(G)$ be the group of all automorphisms of $G$. Following [4], we define $\mathcal{A}(G)=\left\{\alpha \in \operatorname{Aut}(G) \mid x x^{\alpha}=x^{\alpha} x\right.$ for all $\left.x \in G\right\}$ and any element of this set is called a commuting automorphism. This definition first was considered for rings, see [2], [5] and [11]. Also I. N. Herstein in [8] posed a question: when $\mathcal{A}(G)=1$ ? Then T. J. Laffey ([9]) and M. Pettet ([12]) provided extensions of Herstein's result. Moreover we see that $\mathcal{A}(G)$ is a subset of $\operatorname{Aut}(G)$ and $\operatorname{Aut}_{c}(G)$, the group of central automorphisms of $G$ is a subset of $\mathcal{A}(G)$. This observation suggests a question which was considered by Deaconescu, Silberberg and Walls in [4]:

Is it true that the set $\mathcal{A}(G)$ is always a subgroup of $\operatorname{Aut}(G)$ ?

Obviously $\operatorname{Aut}_{c}(G)=\mathcal{A}(G)=\operatorname{Aut}(G)$ when $G$ is abelian. Moreover it is shown in [4] that $\mathcal{A}(G)$ is not always a subgroup of $\operatorname{Aut}(G)$. They constructed a finite non-abelian 2-group $G$ of order $2^{5}$ such that $\mathcal{A}(G)$ is not a subgroup of $\operatorname{Aut}(G)$. In this paper we answer this question in some families of groups. Specially we show that if $G$ is a finite $A C$-group, a finite $p$-group of maximal

2010 Mathematics Subject Classification. 20F28, $20 \mathrm{D} 15$.

Key words and phrases. Commuting automorphisms, $A C$-groups, minimal non-abelian $p$-groups, metacyclic $p$-groups, $p$-groups of maximal class. 
class, or a finite metacyclic $p$-group, then $\mathcal{A}(G)$ is a subgroup of $\operatorname{Aut}(G)$ and in some cases $\mathcal{A}(G)=\operatorname{Aut}_{c}(G)$. We note that a group $G$ is called an $A C$-group if the centralizer of every non-central element of $G$ is abelian. Therefore we deduce that $\mathcal{A}(G)$ is also a subgroup of $\operatorname{Aut}(G)$ when $G$ is a finite minimal non-abelian group, a $p$-group with the central quotient of order less than $p^{4}$, a $p$-group of order less that $p^{5}$ or a finite $p$-group with a cyclic maximal subgroup.

Throughout this paper the following notation is used. All groups are assumed to be finite. The letter $p$ denotes a prime number. $\mathcal{C}_{G}(x)$ is the centralizer of an element $x$ in a group $G$. The nilpotency class of a group $G$ is denoted by $\operatorname{cl}(G)$. A $p$-group of maximal class is a non-abelian group $G$ of order $p^{n}$ with $\operatorname{cl}(G)=n-1$. The terms of the lower central series of $G$ are denoted by $\gamma_{i}(G)$. If $\alpha$ is an automorphism of $G$ and $x$ is an element of $G$, we write $x^{\alpha}$ for the image of $x$ under $\alpha$ and $[x, \alpha]$ is $x^{-1} x^{\alpha}$. Also $\operatorname{Aut}_{Z}^{Z}(G)$ is the group of central automorphisms of $G$, which fix $Z(G)$ elementwise. We write $[a, b]$ for $a^{-1} b^{-1} a b$ when $a, b \in G$. Finally $\mathbb{Z}_{m}^{n}$ is the direct product of $n$ copies of the cyclic group of order $m$.

\section{2. $A C$-groups}

In this section we prove that $\mathcal{A}(G) \leq \operatorname{Aut}(G)$ for any $A C$-group $G$. As a consequence we see that if $G$ is a minimal non-abelian group, a non-abelian $p$ group with $|G / Z(G)| \leq p^{3}$, a $p$-group $(p>2)$ with a cyclic maximal subgroup or a $p$-group of order less that $p^{5}$, then $\mathcal{A}(G) \leq \operatorname{Aut}(G)$. Moreover in some cases we see that $\mathcal{A}(G)=\operatorname{Aut}_{c}(G)$. First we state two following lemmas that are needed for the main results of the paper. $\left[x, y^{\alpha}\right]$

Lemma 2.1 ([4, Lemma 2.1]). If $\alpha \in \mathcal{A}(G)$ and $x, y \in G$, then $\left[x^{\alpha}, y\right]=$

Lemma 2.2 ([4, Lemma $2.4(\mathrm{vi})])$. Let $G$ be a group and $\alpha, \beta \in \mathcal{A}(G)$. Then $\alpha \beta \in \mathcal{A}(G)$ if and only if $\left[x^{\alpha}, x^{\beta}\right]=1$ for all $x \in G$.

Lemma 2.3. If $G$ is an $A C$-group, then $\mathcal{A}(G) \leq \operatorname{Aut}(G)$.

Proof. Let $\alpha, \beta \in \mathcal{A}(G)$. Since $\mathcal{A}(G)$ is finite, it is enough to prove that $\alpha \beta \in \mathcal{A}(G)$ or equivalently $\left[x^{\alpha}, x^{\beta}\right]=1$ for all $x \in G$ by Lemma 2.2. First if $x \in G \backslash Z(G)$, then $\mathcal{C}_{G}(x)$ is abelian and so $\left[x^{\alpha}, x^{\beta}\right]=1$. Also if $x \in Z(G)$, then $x^{\alpha}, x^{\beta} \in Z(G)$, as desired.

Lemma 2.4. Let $G$ be a non-abelian p-group with $|G / Z(G)| \leq p^{3}$. Then $G$ is an $A C$-group.

Proof. Let $g$ be a non-central element of $G$. Then $Z(G)<Z\left(\mathcal{C}_{G}(g)\right) \leq$ $\mathcal{C}_{G}(g)<G$ since $g \in Z\left(\mathcal{C}_{G}(g)\right) \backslash Z(G)$. This implies that $\left|\frac{\mathcal{C}_{G}(g)}{Z\left(\mathcal{C}_{G}(g)\right)}\right|$ divides $p$. Hence $Z\left(\mathcal{C}_{G}(g)\right)=\mathcal{C}_{G}(g)$, as desired. 
Lemma 2.5 ([3, Theorem 1.2]). Let $G$ be a group of order $p^{n}$ with a cyclic maximal subgroup. Then $G$ has one of the following presentations:

(i) $M_{p^{n}}=\left\langle a, b \mid a^{p^{n-1}}=b^{p}=1, a^{b}=a^{1+p^{n-2}}\right\rangle$, where $n \geq 4$ if $p=2$.

(ii) $D_{2^{n}}$, the dihedral group.

(iii) $Q_{2^{n}}$, the generalized quaternion group.

(iv) $S D_{2^{n}}(n>3)$, the semi dihedral group.

COROllary 2.6. For any of the following groups, $\mathcal{A}(G)$ is a subgroup of $\operatorname{Aut}(G)$.

(i) $G$ is a non-abelian p-group with $|G / Z(G)| \leq p^{3}$.

(ii) $G$ is a p-group of order less than $p^{5}$.

(iii) $G$ is a p-group with a cyclic maximal subgroup, where $p>2$.

(iv) $G$ is a minimal non-abelian group.

Proof. (i)-(ii) This follows from lemmas 2.3 and 2.4.

(iii) It is easy to see that $|G / Z(G)|=p^{2}$ by Lemma 2.5 (i). The rest follows from (i).

(iv) This is clear by the fact $G$ is an $A C$-group and Lemma 2.3 .

Lemma 2.7. If $G=M_{p^{n}}$, then $\mathcal{A}(G)=\operatorname{Aut}_{c}(G)$.

Proof. By Lemma 2.5(i), we see that $\mathcal{C}_{G}(a)=\langle a\rangle,\left|G^{\prime}\right|=p$ and $Z(G)=$ $\Phi(G)=\left\langle a^{p}\right\rangle$. Let $\alpha \in \mathcal{A}(G)$, then we may write $a^{\alpha}=a^{i} b^{j}$ and $b^{\alpha}=a^{r} b^{s}$, where $0 \leq s, j<p$ and $0 \leq i, r<p^{n-1}$. Since $\left[a^{\alpha}, a\right]=1$ we deduce that $b^{j} \in \mathcal{C}_{G}(a)$. Hence $b^{j} \in\langle a\rangle \cap\langle b\rangle=1$ and so $(i, p)=1$. Also we have $1=\left[b^{\alpha}, b\right]=\left[a^{r}, b\right]=[a, b]^{r}$ which implies that $p$ divides $r$. Therefore $a^{r} \in Z(G)$. Now by applying [7, Proposition 3, p. 44] and the third relation of the presentation of $G$ we deduce that $1=[a, b]^{i(s-1)}$ and so $p$ divides $s-1$ since $(i, p)=1$. Therefore $s=1$. Moreover by Lemma 2.1, we have $\left[a^{\alpha}, b\right]=\left[a, b^{\alpha}\right]$, which yields that $[a, b]^{i-1}=1$ or equivalently $p$ divides $i-1$. Therefore $\alpha \in \operatorname{Aut}_{c}(G)$, completing the proof.

\section{3. $p$-GROUPS OF MAXIMAL CLASS}

Let $G$ be a $p$-group of maximal class and order $p^{n}$, where $n \geq 4$. In this section we show that $\mathcal{A}(G)=\operatorname{Aut}_{c}(G)$. First we give some properties of $p$-groups of maximal class.

Lemma 3.1. Let $G$ be a p-group of maximal class and order $p^{n}$. Then

(i) $G$ is purely non-abelian,

(ii) $\operatorname{Aut}_{c}(G) \cong \mathbb{Z}_{p} \times \mathbb{Z}_{p}$.

Proof. (i) Assume by the way of contradiction that $G=A \times B$, where $A$ is a non-trivial abelian subgroup of $G$ and $B$ is a purely non-abelian subgroup of $G$. Then $\operatorname{cl}(G)=\operatorname{cl}(B)=n-1$, which is a contradiction since $|B|$ divides $p^{n-1}$. 
(ii) By (i) and $\left[1\right.$, Theorem 1], we have $\left|\operatorname{Aut}_{c}(G)\right|=p^{2}$ since $G / G^{\prime} \cong \mathbb{Z}_{p} \times \mathbb{Z}_{p}$ and $Z(G) \cong \mathbb{Z}_{p}$. Moreover

$$
\operatorname{Aut}_{Z}^{Z}(G) \cong \operatorname{Hom}(G / Z(G), Z(G)) \cong \operatorname{Hom}\left(\frac{G / Z(G)}{(G / Z(G))^{\prime}}, Z(G)\right) \cong \mathbb{Z}_{p} \times \mathbb{Z}_{p}
$$

by $\left[13\right.$, Result 1.1], which completes the proof since $\operatorname{Aut}_{Z}^{Z}(G) \leq \operatorname{Aut}_{c}(G)$.

Let $G$ be a $p$-group of maximal class and order $p^{n}(n \geq 4)$, where $p$ is a prime. Following [10], we define the 2-step centralizer $K_{i}$ in $G$ to be the centralizer in $G$ of $\gamma_{i}(G) / \gamma_{i+2}(G)$ for $2 \leq i \leq n-2$ and define $P_{i}=P_{i}(G)$ by $P_{0}=G, P_{1}=K_{2}, P_{i}=\gamma_{i}(G)$ for $2 \leq i \leq n$. Take $s \in G-\bigcup_{i=2}^{n-2} K_{i}$, $s_{1} \in P_{1}-P_{2}$ and $s_{i}=\left[s_{i-1}, s\right]$ for $2 \leq i \leq n-1$. It is easily seen that $\left\{s, s_{1}\right\}$ is a generating set for $G$ and $P_{i}(G)=\left\langle s_{i}, \ldots, s_{n-1}\right\rangle$ for $1 \leq i \leq n-1$. We note that $P_{n-1}=Z(G)$.

For the rest of this section we fix the above notation.

LEMMA 3.2 ([6, Hilfssatz III. 14.13]). If $G$ is a p-group of maximal class of order $p^{n}$ and $s \notin K_{i}$ for $2 \leq i \leq n-2$, then $\mathcal{C}_{G}(s)=\langle s\rangle P_{n-1}(G)$ and $s^{p} \in P_{n-1}$.

Now we state the following Lemma from [4] that will be used in the sequel. $\mathcal{C}_{G}\left(G^{\prime}\right)$

Lemma 3.3 ([4, Lemma 2.2]). If $\alpha \in \mathcal{A}(G)$ and $x \in G$, then $[x, \alpha] \in$

THEOREM 3.4. Let $G$ be a p-group of maximal class and order $p^{n}$, where $n \geq 4$. Then $\mathcal{A}(G)=\operatorname{Aut}_{c}(G)$.

Proof. Let $\alpha \in \mathcal{A}(G)$, then we may write $s^{\alpha}=s x$ and $s_{1}^{\alpha}=s_{1} y$, where $x, y \in \mathcal{C}_{G}\left(G^{\prime}\right)$ by Lemma 3.3. Now by considering $\left[s^{\alpha}, s\right]=1$ we see that $x \in \mathcal{C}_{G}(s)$ and so we may assume that $x=s^{i} z$, where $z \in Z(G)$ and $0 \leq i<p$ by Lemma 3.2. We claim that $i=0$. Otherwise, since $x \in \mathcal{C}_{G}\left(G^{\prime}\right)$, we have $1=\left[s^{i} z, s_{2}\right]=\left[s^{i}, s_{2}\right]$. Hence $s_{3}=\left[s_{2}, s\right]=1$ since $(i, p)=1$. Therefore $P_{3}(G)=1$ and so $|G|=p^{3}$, which is impossible. Therefore $x \in Z(G)$. Moreover $\left[s^{\alpha}, s_{1}\right]=\left[s, s_{1}^{\alpha}\right]$ by Lemma 2.1, which implies that $y \in \mathcal{C}_{G}(s)$ by using the fact that $y \in \mathcal{C}_{G}\left(G^{\prime}\right)$. Hence by the same argument as above we conclude that $y \in Z(G)$ and so $\alpha \in \operatorname{Aut}_{c}(G)$, as desired.

COROLlary 3.5. Let $G$ be a group of order $p^{n}$ with a cyclic maximal subgroup. Then $\mathcal{A}(G)=\operatorname{Aut}_{c}(G)$.

Proof. If $G=D_{8}$ or $Q_{8}$, then it is easy to check that $\mathcal{A}(G)=\operatorname{Aut}_{c}(G)$. Therefore the proof follows from lemmas 2.5, 2.7 and Theorem 3.4. 


\section{Metacyclic $p$-Groups}

Let $G$ be a non-abelian metacyclic $p$-group. In this section we show that $\mathcal{A}(G) \leq \operatorname{Aut}(G)$. We know that there exists a normal cyclic subgroup $\langle a\rangle$ of $G$ such that $G /\langle a\rangle$ is cyclic. Therefore we may choose an element $b \in G$ and a number $1 \leq k<|a|$ such that $G=\langle b, a\rangle$ and $b^{-1} a b=a^{k}$ and so any element of $G$ has the form $b^{j} a^{i}$ for $j, i \geq 0$.

For the rest of the paper we fix the above notation.

Lemma 4.1. Let $G$ be a non-abelian metacyclic p-group.

(i) $k \equiv 1(\bmod p)$,

(ii) $\left[a^{i}, b\right]=[a, b]^{i}=a^{(k-1) i}$ and $\left[b^{n}, a\right]=[b, a]^{1+k+\cdots+k^{n-1}}$ for $i, n \geq 1$,

(iii) $G^{\prime}=\langle[a, b]\rangle$,

(iv) if $b^{s-1} \in \mathcal{C}_{G}\left(G^{\prime}\right)$, where $s \geq 1$, then $\left[b^{n s}, a\right]=\left[b^{s}, a\right]^{1+k+\cdots+k^{n-1}}$ for any $n \geq 1$.

Proof. (i) Obviously $G^{\prime} \leq\langle a\rangle$ and $\left\langle a^{k-1}\right\rangle \leq G^{\prime}$. Now if $(p, k-1)=1$, then $G^{\prime}=\langle a\rangle$ and so $G / G^{\prime}$ is cyclic which is a contradiction.

(ii) This follows from $b^{-1} a b=a^{k}$.

(iii) We have $G^{\prime}=\langle[x, y] \mid x, y \in G\rangle$ which completes the proof by using

(iv) We use induction on $n$ and the fact that $\left[b^{n s}, a\right]^{b}=\left[b^{n s}, a\right]^{k}$ since $\left[b^{n s}, a\right] \in\langle a\rangle$ and $b^{-1} a b=a^{k}$.

THEOREM 4.2. Let $G$ be a non-abelian metacyclic p-group. Then

$$
\mathcal{A}(G) \leq \operatorname{Aut}(G) .
$$

Proof. Let $\alpha \in \mathcal{A}(G)$, then we may write $a^{\alpha}=a^{i} b^{j}$ and $b^{\alpha}=b^{s} a^{l}$. Therefore $b^{j}, a^{l} \in Z(G)$ by the definition of $\mathcal{A}(G)$. Hence we may assume that $a^{\alpha}=a^{i} z_{1}$ and $b^{\alpha}=b^{s} z_{2}$, where $z_{1}, z_{2} \in Z(G)$. Consequently for $\beta \in \mathcal{A}(G)$ we have $a^{\beta}=a^{i^{\prime}} z_{1}^{\prime}$ and $b^{\beta}=b^{s^{\prime}} z_{2}^{\prime}$, where $z_{1}^{\prime}, z_{2}^{\prime} \in Z(G)$. Now if $g \in G$, then we may write $g=b^{r} a^{t}$. Therefore $\left[g^{\alpha}, g^{\beta}\right]=\left[b^{s r}, a\right]^{i^{\prime} t}\left[b^{s^{\prime} r}, a\right]^{-i t}$ by Lemma 4.1(ii). Moreover by Lemma 3.3, $b^{-1} b^{\alpha}$ and $b^{-1} b^{\beta} \in \mathcal{C}_{G}\left(G^{\prime}\right)$ or equivalently $b^{s-1}, b^{s^{\prime}-1} \in \mathcal{C}_{G}\left(G^{\prime}\right)$. Also by Lemma 2.1 , we see that $\left[b, a^{i}\right]=\left[b^{s}, a\right]$ and $\left[b, a^{i^{i}}\right]=\left[b^{s^{\prime}}, a\right]$. This implies that $\left[g^{\alpha}, g^{\beta}\right]=1$ by Lemma 4.1(iv) and (ii), which completes the proof by Lemma 2.2 .

\section{REFERENCES}

[1] J. E. Adney and T. Yen, Automorphisms of a p-group, Illinois J. Math. 9 (1965), $137-143$.

[2] H. E. Bell and W. S. Martindale, Centralizing mappings of semiprime rings, Canad. Math. Bull. 30 (1987), 92-101.

[3] Y. Berkovich, Groups of prime power order. Vol. 1, Walter de Gruyter, Berlin, 2008.

[4] M. Deaconescu, G. Silberberg and G. L. Walls, On commuting automorphisms of groups, Arch. Math. (Basel) 79 (2002), 423-429. 
[5] N. Divinsky, On commuting automorphisms of rings, Trans. Roy. Soc. Canada. Sect. III. 49 (1955), 19-22.

[6] B. Huppert, Endliche Gruppen. I, Springer-Verlag, Berlin-New York, 1967.

[7] D. L. Johnson, Presentation of groups, 2nd ed., Cambridge University Press, Cambridge, 1997.

[8] I. N. Herstein, J. L. Brenner and W. A. Newcomb, Problems and solutions: elementary problems: E3039-E3040, Amer. Math. Monthly 91 (1984), 203.

[9] I. N. Herstein, T. J. Laffey, and J. Thomas, Problems and solutions: solutions of elementary problems: E3039, Amer. Math. Monthly 93 (1986), 816-817.

[10] C. R. Leedham-Green and S. McKay, The structure of groups of prime power order, Oxford University Press, Oxford, 2002.

[11] J. Luh, A note on commuting automorphisms of rings, Amer. Math. Monthly 77 (1970), 61-62.

[12] M. Pettet, Personal communication.

[13] P. Schmid, Normal p-subgroups in the group of outer automorphisms of a finite pgroup, Math. Z. 147 (1976), 271-277.

S. Fouladi

Faculty of Mathematical Sciences and Computer

Kharazmi University

50 Taleghani Ave., Tehran 1561836314

Iran

E-mail: s_fouladi@tmu.ac.ir

R. Orfi

Department of Mathematics

Faculty of Science, Arak University

Arak 38156-8-8349

Iran

E-mail: r-orfi@araku.ac.ir

Received: 13.8.2012. 